\title{
Improved Field Emission Currents of Carbon Nanotubes after Laser Irradiation
}

\author{
Jungwoo Lee, Jaehong Park, and Whikun Yi \\ Deparment of Chemistrv. Hanvang Lniversitw, Seoul 133-791. Korea. "E-mail: whyighanvang.achr \\ Received March 28, 2006
}

\begin{abstract}
Field emission (FE) currents were measured for silver-pasted and glass-pasted single-walled carbon nanotubes (SWNTs) after illuminating the tubes with a pulsed 532 nu laser. A very low tunt-on field of approximately $0.4 \mathrm{~V} / \mu \mathrm{m}$ and a high current density $-1700 \mu \mathrm{A} / \mathrm{cm}^{2}$ at $3.5 \mathrm{~V} / \mu \mathrm{m}$ was obtained for the silver-pasted SWNTs after laser irradiation but on the whole. no improvements were found for the glass-pasted SWNTs. Two roles of laser irradiation for the silver-pasted SWNTs were proposed. First. the embedded SWNTs and SWNT bundles inside the silver paste were immerged on the outer surface due to an instantaneous melting or amealing of the silver metals by the laser resulting in an increase of the field emission sites. Second, the laser irradiation was thought to improve the electrical contact between SWNTs and the silver metal by reducing the contact resistance via laser-induced thermal annealing. which was responsible for increasing the FE currents.
\end{abstract}

Key Words : Field emission. Carbon nanotube. Laser irradiation. Silver paste. Electrical contact

\section{Introduction}

The field emission (FE) properties of carbon nanotubes (CNTs) are of great importance. especially in applications involving flat panel display devices such as field enission displays (FED). ${ }^{1}$ The electron enitters of the FED must be long-lived and stable. and possess a low turn-on threshold voltage and a high current density at a given extennal field ${ }^{\hat{3}, \hat{3}}$ In general, the enission source should have highly oriented and well-distributed tubes in order to utilize the characteristics of the nanotubes for FE. A notable method using plasma for the synthesis of the aligned CNTs was developed by Ren $e t \mathrm{al}^{+}$This was carried out at a relatively low temperature. which enabled the tubes to be grown on glass. The method involves the initial deposition of a thin nickel layer on the glass followed by the use of this nickel layer as a catalyst to grow nanotubes by plasina enhanced (PE) hot filament chemical vapor deposition (CVD), with acetylene as the carbon source. Unfortunately there are critical difficulties involved with enlarging the electron-enitting area of the device due to the lack of proper equipment and teclunology. Furthermore. this method requires the fabrication of CNT patterns and gate electrodes on the glass substrate to be used as cathode electrodes and to drive FED electrically. respectively. A simple alternative way of fabricating cathode and gate electrodes is to build pattened films using a postdeposition technique. This can be realized either by depositing the CNT films followed by subsequent patterning on the substrate or by growing the CNTs directly on the substrate prepatterned with a catalyst. We have developed a new postdeposition technique which involves mixing purified CNT powder with chemicals and successively screen-printing the mixture onto a substrate. ${ }^{5}$ This has the advantage of enlarging the cathode area of the FED. Recently, we demonstrated a 5.2 -inch. full-color moving picture display using singlewalled carbon nanotubes (SWNTs). ${ }^{6}$ The purified SWNTs were mixed with silver powders and organic binders such as nitrocellulose. and the resulting paste was printed onto an indium tin oxide (TTO)-pattened sodalime glass through a metal mesh. The emitter was subsequently heated in order to remove the organic binders. The remaining paste should be conductive and able to supply electrons from an outside current supplier to the SWNTs upon the departure of field emitted electrons. Furthermore, the paste surface should be polished mechanically to enit sufficient electrons before the next sealing process. ${ }^{5,6}$

For silicon and molybdenum field enitter arrays, a laser has been used as an efficient cleaning technique for decontaminating the field emitter array surfaces, yielding a significant increase in the emission current. ${ }^{78}$ For CNT emitters, especially those deposited on a substrate by postdeposition tecluniques, there is little data established that examines laser treatment. Here, we report the field emission characteristics of SWNTs under the irradiation of laser light which were prepared by a post-deposition technique using a screen-printing method with a silver paste or a glass paste. The effect of laser irradiation on the silver-pasted SWNTs will be explained from the perspective of increasing the number of field emission sites and the electrical contact between the SWNTs and silver metal.

\section{Experimental Section}

Carbon nanotube-containing paste samples were prepared by mixing SWNTs generated from arc discharge with a silver paste or a frit-containing glass paste and organic materials. The composite was printed on a glass substrate and successively heated to $450{ }^{\circ} \mathrm{C}$ to remove organic materials. For FE measurements. a grounded ITO served as an anode above the SWNT-containing cathode plate. which was biased with a negative potential. A homemade $300 \mu \mathrm{m}$ thick alumina spacer was used in between the cathode and the anode and FE currents were monitored with a precise multimeter (Keithley 6517) connected to the cathode plate. 
Pulsed Nd-Yag laser (Quantel, Brilliant) was used to illuminate the SWN $\mathrm{N}$-containing paste samples with a wavelength of $532 \mathrm{~nm}$. which corresponds to the second harmonics of original infrared light (1064 nm). The repetition rate of the laser was $50 \mathrm{~Hz}$, the intensity of the light was varied from $1 \mathrm{~mJ} /$ pulse to $20 \mathrm{~mJ} /$ pulse per $\mathrm{cm}^{2}$, and the measured pulse width was $5.6 \mathrm{nsec}$.

\section{Results and Discussion}

Figure 1 shows the relationship between the FE currents and the intensity of the $532 \mathrm{~nm}$ pulse laser for the silverpasted and glass-pasted SWN' samples. As the laser intensity increased from 0 to 4.2 and $18 \mathrm{~mJ} /$ pulse per $\mathrm{cm}^{2}$. the FE currents increased for the silver-pasted sample. On the other hand, the $F E$ currents were not affected for the glass-pasted sample but rather decreased upon the repeated shooting of laser pulses. The turn-on field $\left(\mathrm{E}_{10}\right)$, which is defined as the field required to generate an emission current density of $10 \mu \mathrm{A} / \mathrm{cm}^{2}$ measured from figure $\mathrm{I}(\mathrm{a})$ is $2.6 \mathrm{~V} /$ $\mu \mathrm{m}$ before lasing. After exposure to the laser and increasing the laser intensity up to 4.2 and $18.3 \mathrm{~mJ} /$ pulse, the $\mathrm{E}_{\mathrm{to}}$ for the silver-pasted SWN'S decreased to 0.8 and $0.4 \mathrm{~V} / \mu \mathrm{m}$, respectively. From our repeated experiments, the $E_{10}$ was found to be in the range of $0.4-0.7 \mathrm{~V} / \mu \mathrm{m}$ with an average
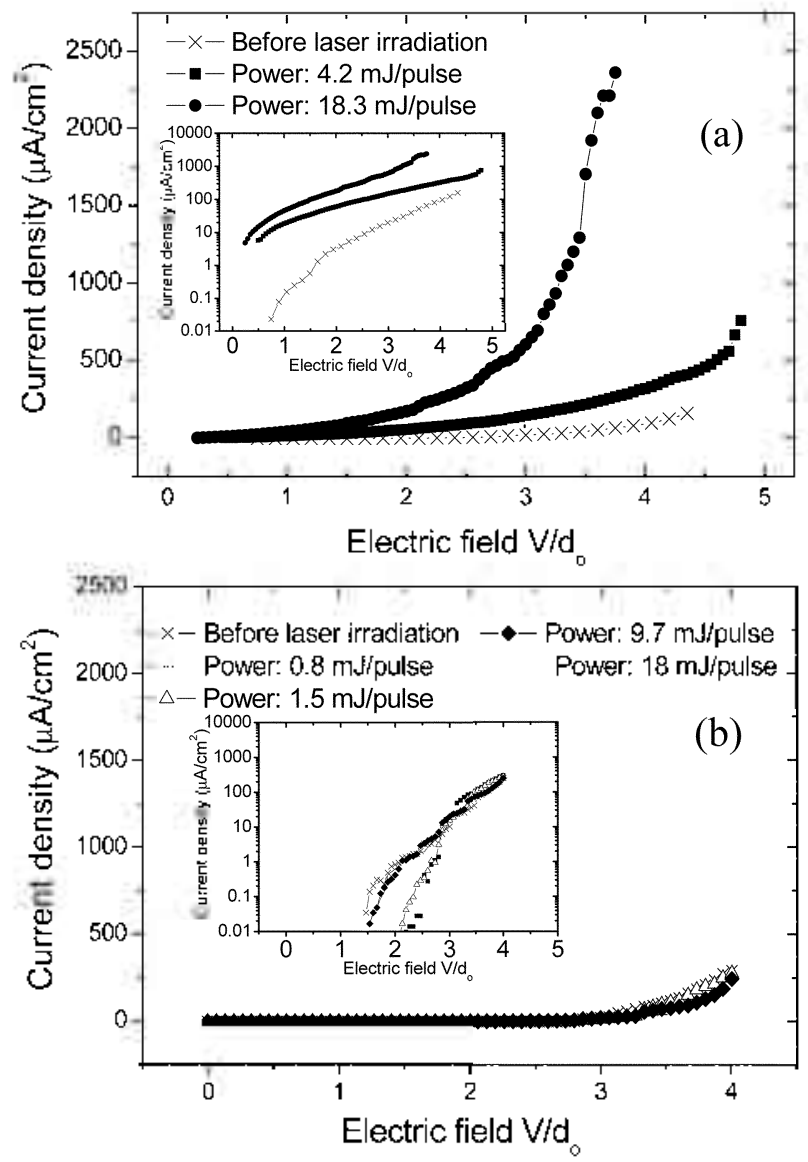

Figure 1. Field emission characteristics of (a) silver-pasted SWNTs and (b) glass-pasted SW.VIs with respect to various laber intemsitics at $532 \mathrm{~nm}$. value of $0.6 \mathrm{~V} / \mu \mathrm{m}$ at the intensity of an $18 \mathrm{~mJ} / \mathrm{pulse}$ after 1500 pulses by the laser (i.e.. 50 repetition rate $/ \mathrm{sec} \times 30 \mathrm{sec}$ ). This value is remarkably low compared with the reported values of 1.5 and $2.1 \mathrm{~V} / \mu \mathrm{m}$ obtained from the SWN' films by Refs. 9 and 10, respectively. Furthermore, when we compare the emission current of Figure $\mathrm{l}(\mathrm{a})$ at the $3.5 \mathrm{~V} / \mu \mathrm{m}$ with the data from Ref. 9, our value, $\cdots] 700 \mu \mathrm{A} / \mathrm{cm}^{2}$, is about three times higher than reported previously. ${ }^{9}$ However, when the laser intensity was increased to over $18 \mathrm{~mJ} /$ pulse, the FE currents decreased from the highest values. An examination of the sample with scanning electron microscopy (SEM) indicated a disappearance of the SWN'S in the area exposed to the laser. This showed that laser treatment over a certain limit of light intensity induced the laser ablation of the SWN'l's from the pasted samples.

lo determine the laser irradiation effect in Figure l(a). SEM pictures taken before and after laser exposure were examined. As shown in Figure 2(a), in the case of the silverpasted sample, a few of the SWN'ls could be seen in the SEM picture taken before laser irradiation. However, after exposure to laser light. many more $\mathrm{SWN}$ 's and $\mathrm{SWN}$ '] bundles were found in the affected area (Fig. 2(b)). This implied that the embedded SWN's and SWN'T bundles

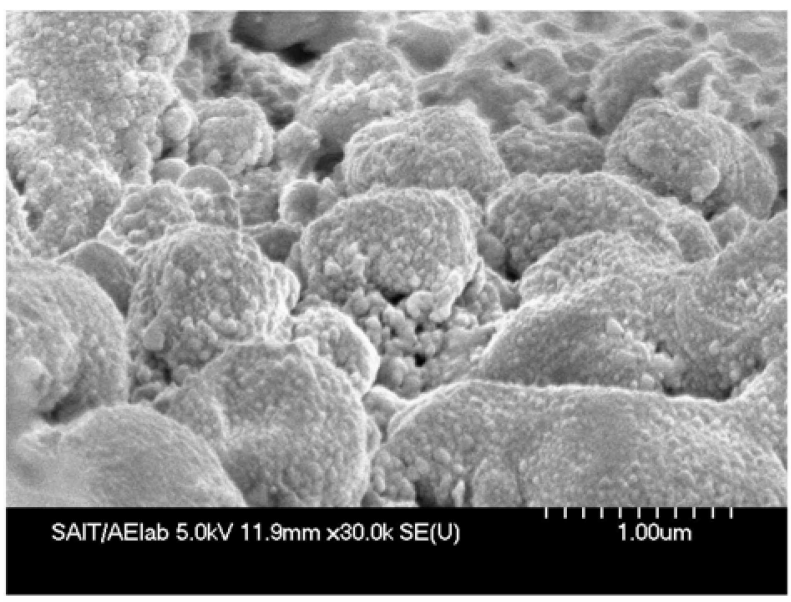

(a)

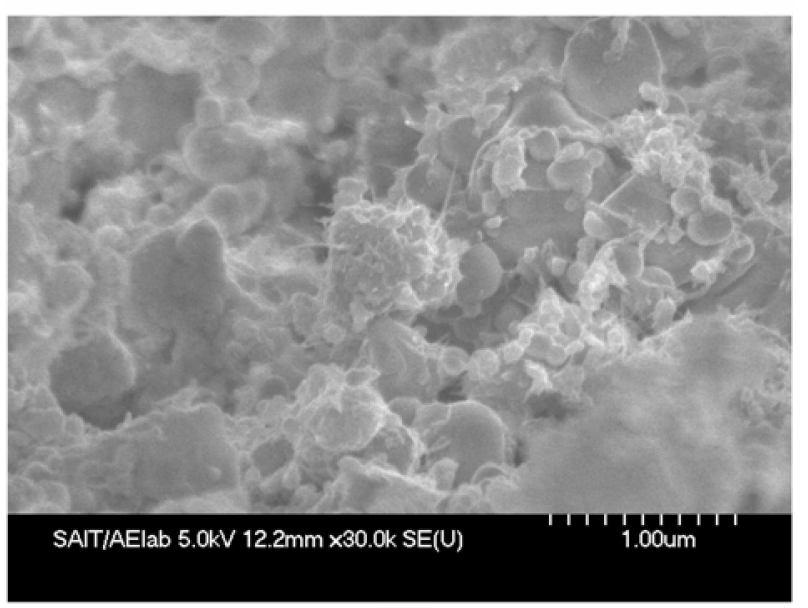

(b)

Figure 2. SEM pictures of silver-pasted SWVTs (a) before and (b) alter the laser irradiation at $532 \mathrm{~nm}$. 


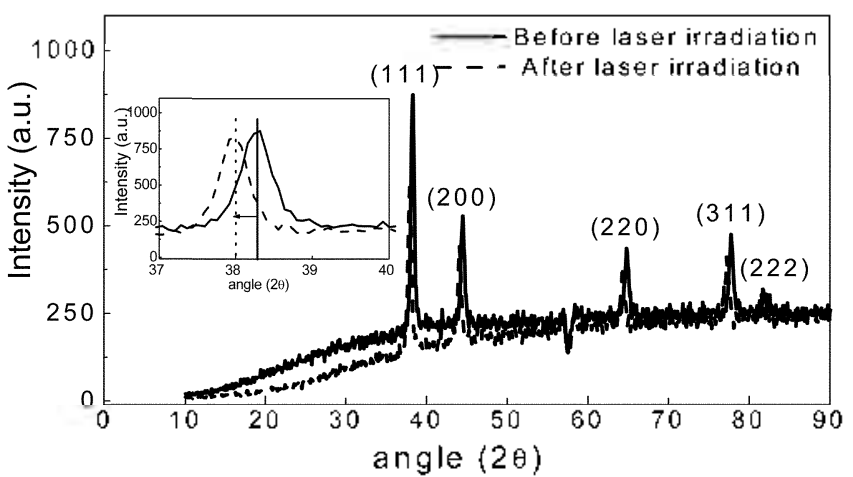

Figure 3. XRI) spectra of silver-pasted SWNTs before and after laser irradiation. The inset represents a shift of the $\mathrm{Ag}(111)$ peak after lasing.

inside the silver paste were exposed on the outer surface during laser irradiation. which was responsible for increasing the f'E currents. The laser pulse seemed to inelt the silver metal slightly at the given experimental conditions. To understand the effect of pulsed laser irradiation, X-ray diffraction (XRD) spectra of the silver-pasted SWNT samples were also measured before and after laser irradiation, for comparison (Figure 3). XRD peaks were located around $2 \theta=38,44,64,77$, and 81 degrees for both cases, which implied a face-centered cubic silver crystalline structure. However, all of the $2 \theta$ values shifted to lower positions by $0.3-0.4$ degrees after the laser treatment. The inset of Figure 3 shows such a behavior in the case of $2 \theta=38$ degrees. In other words, each $2 \theta$ value after laser irradiation approached the positions reported by the Natl. Bur. Stand." This strongly suggested that the silver pasted SWNT' sample was in a high strain andior stress environment initially; however, the strain and/or stress was released after laser irradiation due to an instantaneous melting of the silver metal.

When we applied laser irradiation to the glass-pasted SWNT's, the FE current was not increased with any length of exposure time, rather it decreased upon repeated laser shooting (see the fig. I(b)). This result corresponded with our SEM observations (not shown) that glass beads inside the glass paste were not changed after irradiation by a low intensity laser, however, they were destroyed and disappeared upon repeated shooting with a high intensity pulsed laser. The main component in glass is silica, $\mathrm{SiO}_{2}$, and it is well known this material does not absorb $532 \mathrm{~nm}$ photons, thus the glass-pasted SWNI sample cannot interact with the laser light.

For applications in an HED device with a post deposition technique which uses silver-pasted SWNT's, for example, the carbon nanotubes must be connected with conductive substrates (in this case, silver metal) with low contact resistance in order to serve as a good cathode. Several attempts have been made to improve the electrical contact of $\mathrm{CNIs}$, including selective electron bombardment of the contact area by introducing defects in the $\mathrm{CN} / \mathrm{s},{ }^{12}$ thermal annealing by forming metal carbide interface layers between the $\mathrm{CNT}$ 's

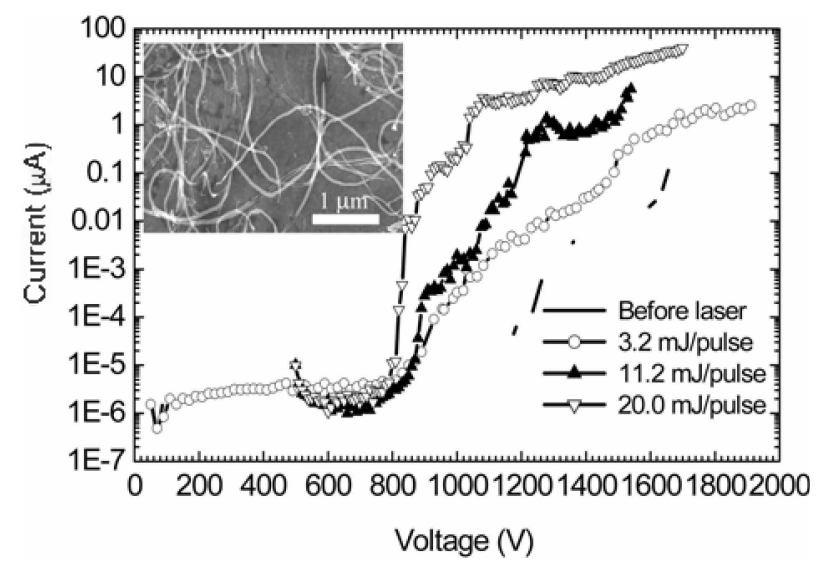

Figure 4. Current-voltage (I-V) characteristics of dispersed CNTs on $\mathrm{Ag}$ film at various intensities of laser light per $\mathrm{cm}^{2}$. Here. a scries of positive voltages ranging from 0 to $2000 \mathrm{~V}$ was applicd to the anode plate (gold-covered indium-lin-oxide (ITO) or ITO itself) with a space separalion of $430 \mu \mathrm{m}$ from the electronically grounded. Lathodie dispersed C N from the dispersed CNTs was collected on the anode. where the open area exposed to the anode was $8.5 \times 8.5 \mathrm{~mm}^{2}$.

and metal, ${ }_{1.3}^{13}$ or dipping the CNTs into liquid $\mathrm{Hg} .{ }^{14}$ We hypothesized that laser irradiation improved the electrical contact between the $\mathrm{CN}$ Is and silver metal. To prove this. the FE currents of the CNT's dispersed on a thin Ag film were measured before and after laser irradiation. For the experiment, a silver $(\mathrm{Ag})$ filln was deposited onto a titaniumcoated $\mathrm{SiO}_{2}$ substrate by magnetron sputtering. where the thickness of the Ag metal layer was maintained at $100 \mathrm{~nm}$. The CNI-dispersed ethanol solution droplets were then dropped on the Ag inetal substrate and dried for $1 \mathrm{hr}$ at 200 " $\mathrm{C}$ to remove the ethanol.

Figure 4 shows the fte curves for the sample prepared by the above method before and after exposure to a $532 \mathrm{~nm}$ laser at various light intensities. The overall amount of the FE current after laser irradiation was quite satisfactory considering the fact that most of the CNIs were lying on the substrate. As can be seen from the inset of Figure 4, the initial layout of the $\mathrm{CN}[\mathrm{s}$ on the silver surface appears unstable from the perspective of electrical contact. The initial fE currents in rigure 4, before lasing, strongly reflected this CN' layout. The dispersed CN's are thought to interact with the silver surface only through van der Waals forces. Therefore, in this case, the effect of laser irradiation could be interpreted as improving the electrical contact between the CNTs and the surface of the silver metal. It is believed that the slight melting of the metal surface due to the heat transfer from the laser leads to an improvement of the electrical contact between the $\mathrm{CN}^{\prime}$ 's and silver surface. This enhances the supply of electrons to the CNIs during the field emission process.

In conclusion, t'E characteristics were examined for silver-pasted and glass-pasted SWNT's upon irradiation with a $532 \mathrm{~nm}$ laser. We obtained a remarkably low turn-on field $\left(\mathrm{E}_{\mathrm{iv}}\right)$ with values such as $0.4 \mathrm{~V} / \mu \mathrm{m}$ and a high current density of approximately $1700 \mu \mathrm{A} / \mathrm{cm}^{2}$ at an electric field of 
$3.5 \mathrm{~V} / \mu \mathrm{m}$ for the silver-pasted SWNTs after laser irradiation below a laser intensity of $20 \mathrm{~mJ} /$ pulse per $\mathrm{cm}^{2}$. Based on the analysis of SEM and XRD data. it was revealed that the embedded SWNTs and SWNT bundles inside the silver paste become more exposed on the outer surface during laser treatment. and this was responsible for increasing the $\mathrm{FE}$ currents. The other important role of the laser treatment was an improvement of the electrical contact between the CNTs and silver metal which was achieved by a reduction of the contact resistance through laser-induced thermal annealing. This improvement of the electrical contacts allowed enough electrons to be supplied to the CNTs during the field emission process.

Acknowledgements. This work was supported by the research fund of Hanyang University and "Ministry of Education and Human Resources Development" sponsoring "The Second Stage of BK2I".

\section{References}

1. De Her. W. A.: Chatelain. A.: Ugaart. D. Science 1995. 270. 1179.

2. Saito. Y: Hamaguchi. K.: Uemura. S.: Uchida. K.: Tasaka. Y: Yumura, M: Kasulya. A.: Nishina, N. Appl. Plys. A: Aater Sci. Process 1998. 67.95.

3. Dean, K. A. Chalamala, B. R. Appl. Phns. Lett 1999. 75, 3017.
4. Ren. Z. F.: Huang. Z. P.; Xu, J. W.: Wang, J. H.: Bush, P.; Siegal. M. P.: Provencio. P. N. Science 1998. 282. 1105.

5. Lee. N. S.: Chung. D. S.: Hant. I. T.: Kang. I. H.: Choi. Y. S.: Kim. H. Y: Park. S. H.: Tin. Y. W.: Y1. W. K.: Yun. M. J.: Jun1g. J. E.: Lee. C. J.: You, J. H., Jo, S. H.: Lee. C. G.: Kim. J. M. Dianond Relat. Hater: 2001. 10. 265.

6. Jung. J. E.: Jin. Y. W.: Choi, J. H.: Park. Y. J.: Ko. T. Y.: Chung. D. S.: Kiml. T. W.: Jan1g. T. E.: Cha. S. N.: Yi. W. K.: Cho. S. H.: Ycont. M. T.: Lee. C. G.: You. J. H.: Lee. N. S.: Yoo. J. B.: Kimn. I. M. Plnsica B 2002.323.71

7. Kanazawa. Y.: Sawada, A. W.: Zhao, J.: Jarupoonphol. W.: Takai. M: Jap. J. Appl. Plys. 2003. 42. 4041

8. Seo. D. S.: Kim, C. O.: Hong, J. P. Shin. J. S.; Song. B. K.: Cha. S. N.: Chung. J. W. Kim. T. M. Appl Phys. Lett 2003. 82. 3299.

9. Bonard. I. M.: Salvetat. T. P.: De Heer. W. A.: Forro. L.: Chatelain. A. Appl. Ply's. Lett. 1998, 73. 918.

10. Obraztsova, E. D; Bonard, J. M: Kuznetsov, V. L.: Zaikovskii, V. I.: Pimenov, S. M.; Pozarov. A. S.: Terekhov, S. V.: Konov. V. I.; Obraztsova. A. N.: Volkov. A. S. Nanostructured Mater 1999. 12 567.

11. Swanson. T.: Tatge. A. Natl Bit: Stand (TS.) Cin. 1953. 539. 23

12. Bachtold. A., Henny, M: Terrier. C.: Strunk. C.: Schoenenberger. C.: Salvetat, J.-P: Bonard, J. M.: Forro, L. Appl. Phws. Lett 1998. 73.274 .

13. Zhang. Y.: Ichihashi. T.: Landree. E.: Nihey: F.: Iijima. S. Science 1999. 285,1719 .

14. Frank. S.: Poncharal. P.: Wang, Z. L.: De Heer, W. A. Science 1998. 280,1744 . 